\title{
Trayectorias personales de aprendizaje y currículo flexible: la perspectiva de los estudiantes universitarios de psicología
}

\author{
Frida Díaz-Barriga Arceo, José-Luis López-Ramírez y Edmundo-Antonio López-Banda
}

\section{RESUMEN}

El objetivo del estudio es desvelar el proceso de construcción de trayectorias personales de aprendizaje y la perspectiva estudiantil respecto al currículo flexible. Se empleó una metodología cualitativa fenomenológica y se entrevistó a 20 estudiantes y egresados de una licenciatura en psicología. Los resultados evidencian que los estudiantes se inclinan por un currículo flexible que les permita preespecializarse conforme a sus intereses respecto a diversos campos de intervención y paradigmas disciplinares de su preferencia. Rechazan trayectorias preestablecidas y obligatorias que desde su perspectiva trastocan su plan de carrera.

Palabras clave: flexibilidad curricular, trayectorias personales de aprendizaje, actores del currículo, estudiantes universitarios, enseñanza de la psicología, México.

Frida Díaz-Barriga Arceo

\section{José-Luis López-Ramírez}

\section{Edmundo-Antonio López-Banda}




\section{Trajetórias pessoais de aprendizagem e currículo flexível: a perspectiva dos estudantes universitários de psicologia}

\section{RESUMO}

O objetivo do estudo é desvelar o processo de construção de trajetórias pessoais de aprendizagem e a perspectiva estudantil sobre o currículo flexível. Se utilizou uma metodologia qualitativa fenomenológica e se entrevistou a 20 estudantes e formandos de uma licenciatura de psicologia. Os resultados evidenciam que os estudantes se inclinam por um currículo flexível que lhes permita pré-especializar conforme seus interesses sobre diversos campos de intervenção y paradigmas disciplinares de sua preferência. Rejeitam trajetórias pré-estabelecidas e obrigatórias que em sua perspectiva pioram seu plano de carreira.

Palavras chave: flexibilidade curricular, trajetórias pessoais de aprendizagem, atores do currículo, estudantes universitários, ensino da psicologia, México.

\section{Personal learning trajectories and flexible curriculum: the university psychology students' perspective}

\section{ABSTRACT}

The objective of the study is to reveal the process of constructing personal learning trajectories and the student's perspective regarding the flexible curriculum. A phenomenological qualitative methodology was used and 20 students and graduates of a degree in psychology were interviewed. The results show that the students are inclined towards a flexible curriculum that allows them to pre-specialize according to their interests in different fields of intervention and disciplinary paradigms they have chosen. They reject pre-established and obligatory trajectories that, from their perspective, disrupt their career plan.

Key words: curriculum flexibility, personal learning trajectories, curriculum actors, university students, psychology teaching, Mexico. 


\section{Introducción}

La noción de "trayectoria" es un instrumento analítico enfocado a entender las actividades de aprendizaje en el tiempo-espacio, así como la conformación de la identidad del aprendiz como una capacidad para interpretar y utilizar los recursos del entorno a través de formas de acción concretas. El concepto de trayectoria personal de aprendizaje (TPA) es importante para comprender la manera en que los sujetos llegan a trazar su propio itinerario para satisfacer sus necesidades de aprendizaje y formación, tanto en escenarios formales como informales. Para Barron (2010) la TPA permite identificar las rutas de aprendizaje por las que pasa una persona que manifiesta un interés amplio y sostenido por actividades que involucran el aprendizaje de alguna capacidad o temática en particular, que tiene la meta de arribar a un conocimiento más o menos profundo de la misma, en el marco de la participación en comunidades de práctica dentro y fuera de la educación formal. Definir una TPA implica que el estudiante requiere de establecer un compromiso (engagement) por determinados aprendizajes en diferentes escenarios y tiempos, disponiendo de información, recursos y redes de apoyo pertinentes que permitan el logro de dicha trayectoria prevista.

Hablar de trayectoria de aprendizaje implica referirse a un recorrido, un camino en construcción permanente y que el concepto va mucho más allá de la idea mecánica de acumulación de grados o certificados escolares, regulados por un trayecto estandarizado en un programa o plan de estudios. Es decir, una visión reduccionista de la trayectoria de aprendizaje de un estudiante se limita a consignar "la sumatoria de sus pasos por los distintos niveles del sistema" (Nicastro y Greco, 2009: 24) porque esto expresa un perfil tipificado y prescrito, no aclara de quién se trata, dónde, con quién o con qué sentido.

Por el contrario, cuando se habla de trayectoria de un estudiante (o de un docente, de un profesional), hay que pensar en un itinerario en situación, en el marco de una historia, de procesos acotados en el interjuego de espacio y tiempo. Es decir, son importantes los motivos, los acontecimientos, el devenir de la persona, para entender quién es él o ella, el sentido de su propio recorrido, su relación con el colectivo del que forma parte. En todo caso, se abre la puerta al cuestionamiento de la distancia entre la trayectoria "ideal", la que ha sido pensada para todos o prescrita por las instituciones formadoras en el proyecto curricular, frente al eventual dinamismo y concreción de las trayectorias reales de los aprendientes.

En este sentido, resaltan el papel de la agencia del propio sujeto como profesional en formación, pero también del contexto institucional donde se forma, de las restricciones y facilidades que impone al trayecto del estudiante. Se trata de procesos de formación que ocurren a través de la interacción con determinados agentes educativos y que promueven la adquisición de saberes y prácticas socioculturales propias de una profesión. Las trayectorias personales de aprendizaje se vinculan tanto con los procesos subjetivos e intersubjetivos de construcción de la identidad, como con el ethos de las instituciones educativas y socializadoras en que participa la persona en formación.

Por otro lado, el estudio de las TPA de los estudiantes nos permite un acercamiento a la dinámica de la comunidad de práctica donde se forman (Wenger, 2001), en este caso de una entidad universitaria específica, del papel que juega en ella y de la manera en que se pretende que se apropie de los saberes de la profesión. El currículo formal, como marco de referencia institucional de los fines y contenidos de la formación del futuro profesional, puede contrastarse con la forma en que se gestiona y opera en la institución, lo que permite, al menos en parte, explicar los logros y los obstáculos que enfrentan los actores del currículo.

Las primeras experiencias de currículo flexible en nuestro país datan de la década de los setenta del siglo XX. Se afirma que el proceso de 
departamentalización y la flexibilidad en los planes de estudio comienzan a debatirse en el campo curricular a partir de la publicación del artículo de Meneses (1971) y su implantación formal en la Universidad Iberoamericana. De manera ideal, el esquema de departamentalización y la posibilidad de optar por determinadas asignaturas, ofrece la ventaja de desarrollar un plan personal de estudios, integrarse en determinadas líneas de investigación con el acompañamiento de expertos en la disciplina y la posibilidad de acceder a un practicum y a actividades de servicio fuera del recinto universitario. Como desventajas, se han mencionado la posibilidad de atomizar el plan de estudios y la existencia de disputas o la falta de comunicación y equidad entre las academias departamentalizadas. Por su parte, Martín (1998) indica que las primeras universidades en el país en acoger los modelos de departamentalización, modelados en las universidades norteamericanas con una orientación profesionalizante, fueron las principales universidades privadas: la Universidad Iberoamericana, el Instituto Tecnológico de Monterrey y la Universidad Autónoma de Guadalajara. En el caso de las universidades públicas, son la Universidad Nacional Autónoma de México (UNAM) y la Universidad Autónoma Metropolitana quienes adoptan el modelo departamental, aunque en cada institución la concreción de la departamentalización, la flexibilidad curricular y la conformación de comunidades académicas es muy distinta. No obstante, la tradición de planes y programas de estudio lineales, inflexibles, será desde entonces motivo de muchas críticas. Los cambios mencionados buscaban formas de gobierno más horizontales "en contraposición a las formas dictatoriales tradicionales" en las universidades: "La flexibilidad del currículum se hace indispensable, así como la participación activa del estudiantado, ya que éste es considerado como responsable directo de su formación" (Martín, 1998: 12).

En el caso que nos ocupa, la creación de la Facultad de Psicología de la UNAM en la primera mitad de los setenta conduce a la departamentalización mediante la conformación de unidades académico-administrativas que albergan comunidades académicas abocadas a docencia e investigación ("departamentos") que con relativa autonomía plantean un tramo curricular flexible en los últimos semestres de la licenciatura. Se conforman desde entonces diversas áreas profesionales en el currículo, a fin de que los estudiantes puedan elegir distintas asignaturas, propias de campos específicos del saber y la práctica profesional (psicología clínica, laboral, social, educacional, experimental, entre otras). No obstante, el título de licenciatura se sigue otorgando con carácter general (licenciatura en psicología), pero todo estudiante puede optar por preespecializarse en al menos un campo de su interés. En el ajuste curricular de 2008 tales áreas prevalecen en la forma de campos de conocimiento e intervención profesional, ligadas a distintas tradiciones psicológicas. Puede decirse que en este caso, como en muchos otros, la estructura académico-administrativa de las facultades y escuelas en las universidades guarda un relativo isomorfismo con la estructura curricular, aunque ello no ha impedido una diversidad de tensiones, tanto académicas como de gestión o prevalencia de determinados grupos de poder.

A partir de la década de los noventa, el tema de flexibilidad curricular como tendencia propia de la globalidad, pasa a formar parte del discurso de los modelos educativos que promulgan un diseño curricular por competencias, centrado en el aprendiz, que privilegia la enseñanza mediante métodos de indagación y experienciales, en una diversidad de escenarios formales e informales y con un fuerte soporte en las tecnologías digitales (Loveless y Williamson, 2017). En estrecho vínculo con el concepto de personalización del aprendizaje ante una nueva ecología del conocimiento (Barron, 2010; Coll, 2016), el currículo flexible se convierte en un imperativo, así como la necesidad de que los aprendices sean capaces de conformar trayectorias personales de aprendizaje, tanto en sus estudios formales como 
en un sinfin de experiencias de aprendizaje a lo largo y ancho de la vida. Sin embargo, en las universidades los procesos de gestión institucionales, los intereses de los grupos de poder, la carencia de formación o condiciones para la docencia innovadora, la falta de habilidades para el estudio independiente de parte de los estudiantes, entre otros factores, pueden operar en contra de los eventuales beneficios de los modelos curriculares innovadores y flexibles.

La flexibilidad curricular debe ser entendida como una adaptación a la acción educativa para ofrecer al estudiante opciones de qué, cuándo y cómo se quiere aprender. Es decir, parte del supuesto de un sujeto con capacidad y agencia para tomar decisiones respecto a la construcción de su proyecto de vida y formación. La flexibilidad en el nivel universitario implica una apertura ordenada que permite generar diversos perfiles de egreso para las distintas ramas profesionales que se pretenden formar. Esto requiere de un currículo centrado en el estudiante, que debe tener la apertura suficiente para elegir las asignaturas o módulos que quiera cursar, así como los docentes con quienes se quiere formar, debiendo aportarse al alumnado la información y apoyos requeridos para la toma de decisiones (Escalona, 2008).

Díaz-Villa (2008) menciona que la flexibilidad curricular puede plantearse por lo menos de dos formas: la primera referida a la apertura de límites y de las relaciones entre campos, áreas y contenidos del currículo; y la segunda, en función del grado de apertura de los cursos o las actividades académicas de acuerdo con las necesidades e intereses de los estudiantes. A su vez, Escalona (2008: 150) afirma que la institución escolar tiene el compromiso de promover "el diseño de una estructura curricular flexible que implica seleccionar, organizar y distribuir los contenidos, lo cual ha de hacerse tomando en cuenta su relevancia, pertinencia, utilidad social e individual, contextualización, aplicabilidad e impacto".

Poner en práctica un proyecto curricular y darle concreción en un proceso formativo que avizora el futuro de una profesión es algo que atañe a toda la comunidad y su gestión no depende exclusivamente de los directivos o del personal académico-administrativo, dado que la transformación de mentalidades y prácticas ocurre principalmente en la interacción entre los agentes educativos, los estudiantes y el contexto de referencia. Debido a ello, desde hace algunos años ha tomado impulso el estudio de los actores del currículo, línea de investigación en la que se busca dar voz a los mismos para recuperar su experiencia en torno a los proyectos curriculares, las reformas educativas y las innovaciones previstas en la enseñanza universitaria (Barrón y Díaz-Barriga, 2016). El caso del currículo flexible y la posibilidad de generar trayectorias personales de aprendizaje ha sido discutido en lo que atañe al diseño y desarrollo del currículo, las políticas educativas y la gestión institucional, pero falta una mayor comprensión de cómo se lleva a la práctica desde la perspectiva de los propios estudiantes, cuestión que se aborda en el presente trabajo.

\section{Método}

\section{Planteamiento del problema}

El cometido de este estudio ha sido comprender cómo es que los estudiantes de la licenciatura en Psicología construyen una trayectoria personal de aprendizaje (TPA) en el tramo formativo flexible del currículo, que abarca del $5^{\circ}$ al $8^{\circ}$ semestre, que es donde eligen las asignaturas que los conducen a la formación profesional. Se trata de estudiantes que cursan o cursaron el plan de estudios 2008, cuya estructura y organización se basa en una visión multiparadigmática y plural de la Psicología, que obedece a distintas tradiciones psicológicas y a campos de conocimiento e intervención profesional, con sus propias teorías, métodos y modelos de intervención. Asimismo, se trata de un currículo que plantea una oferta educativa amplia de asignaturas agrupadas en campos de conocimiento (psicología clínica, social, educativa, neurociencias, etcétera), de manera tal que el estudiante puede conformar su TPA libremente, 
eligiendo asignaturas optativas de su interés, sin más restricción que el cumplimiento de los créditos requeridos en los tiempos curriculares previstos. Para apoyar al estudiante en el proceso de decisiones conducente a conformar su propia TPA, se sugiere en la propuesta curricular original que cada estudiante tenga acceso a tutoría personalizada, asesoría grupal o disponga de una base de información amplia, que la institución requiere poner a su disposición. Cabe aclarar que la primera parte de la formación, que dura cuatro semestres, es de carácter obligatorio y común a todos los estudiantes y pretende darles los conocimientos generales y básicos que debe poseer todo psicólogo (Plan de Estudios de la Licenciatura en Psicología, 2008, UNAM). ${ }^{1}$

En la entidad académica de interés, como en muchas más, no se ha analizado desde la voz de los principales interesados, los estudiantes, la manera en que conforman sus trayectorias en la licenciatura, su capacidad para decidir su propio proyecto formativo y para la profesión, ni las ventajas o limitaciones que encuentran en un plan de estudios flexible. Algunos directivos han expresado que los estudiantes no saben qué ni cómo elegir las asignaturas que cursan y que sería deseable un currículo prescrito, conformado de trayectorias preestablecidas con asignaturas obligatorias. Desde la perspectiva de Rudduck y Flutter (2007) la autoridad tiende a representarse a los estudiantes (niños y jóvenes) como personas inmaduras, carentes de agencialidad y por ello en muchas ocasiones no se les toma en cuenta en decisiones que los afectan directamente. De acuerdo con Torres (2011) lo anterior contraviene el principio de justicia curricular, ya que limita el acceso a programas, contenidos y estrategias educativas diversificadas que operen con base en principios de equidad y se ajusten a necesidades e intereses de los estudiantes, partiendo de que lo que los caracteriza es la diversidad, no la uniformidad.

Con base en estas consideraciones, en esta investigación de tipo cualitativo fenomenológico se procedió a encontrar evidencia con relación a las siguientes interrogantes:

- ¿Cómo conforman los alumnos una TPA en la licenciatura en Psicología en el tramo curricular flexible de elección de asignaturas correspondiente al Área de Formación Profesional $\left(5^{\circ} \mathrm{a}\right.$ $8^{\circ}$ semestres)?

- ¿Con base en qué criterios y tipo de información toman decisiones los alumnos respecto a sus TPA?

- ¿Qué factores favorecen u obstaculizan la construcción de una TPA desde la perspectiva y experiencia de los estudiantes de psicología?

- ¿Qué ventajas y desventajas encuentran los estudiantes entre una trayectoria curricular flexible elegida por ellos y otra obligatoria y preestablecida por las autoridades del plantel?

El aporte del trabajo es comprender desde la voz de los actores del currículo, los estudiantes, cuál ha sido su experiencia y cómo perciben los mecanismos mediante los cuales conforman sus trayectorias personales de aprendizaje durante la formación profesional universitaria. Asimismo, resulta de interés saber qué cuestiones facilitan o dificultan la conformación de su TPA y qué cambios proponen que haga la institución educativa respecto a éstas.

\footnotetext{
${ }^{1}$ Es importante acotar lo que la legislación universitaria de la UNAM (2015) plantea respecto a la finalidad de los planes de estudio de licenciatura, donde se establece que el estudiantado desarrollará su propio proyecto formativo: "En la licenciatura brindar conocimientos que les permitan a los alumnos abordar los problemas con un enfoque disciplinario, interdisciplinario, multidisciplinario o transdisciplinario y que promuevan su formación científica, humanística, artística y ética para su desarrollo cultural y profesional. Dichos estudios tienen como finalidad proporcionar a los alumnos conocimientos, habilidades y métodos de trabajo, así como actitudes y aptitudes relativas al ejercicio de una profesión. Asimismo, ofrecerles las herramientas con el fin que desarrollen su propio proyecto para el ejercicio independiente de la profesión o, en su caso, continúen con estudios de posgrado, y en los estudios técnicos de carácter optativo, obtengan los conocimientos prácticos específicos como eje de la formación" (Capítulo 3, artículo 9 del Reglamento General para la presentación, aprobación, evaluación y modificación de planes de estudio, UNAM, 2015, cursivas nuestras).
} 


\section{Tipo de estudio}

Se condujo un estudio fenomenológico-interpretativo (Tójar, 2006), donde se aborda el estudio de los fenómenos de interés desde la perspectiva (experiencia y significado) de los actores sociales. En este caso, se buscó desvelar y comprender los sentidos y significados de los participantes, estudiantes universitarios de psicología, a partir de su propia experiencia en la licenciatura en Psicología de la UNAM, particularmente en el tramo de formación profesional, respecto al fenómeno de la flexibilidad curricular y personalización de trayectorias. Es un estudio que no busca la representatividad ni la generalización de hallazgos a toda la población estudiantil, sino la comprensión del objeto de estudio desde la voz de los actores, tomando en cuenta la variabilidad de situaciones y condiciones reportadas por los estudiantes, que son la población-meta de toda modificación curricular. Es un estudio que forma parte de la línea actores del currículo, sobre la cual ya se han publicado otros trabajos con estudiantes de esta entidad académica respecto a sus experiencias en el currículo de la licenciatura (Díaz Barriga, Soto y Díaz, 2015; Díaz Barriga, Pérez y Lara, 2016).

\section{Participantes}

Se trata de una muestra intencional que integra 20 estudiantes que cursaban o habían cursado el plan de estudios 2008 de la licenciatura en Psicología, UNAM, en las generaciones 2010 a 2016, modalidad presencial. En el momento del estudio (semestre 2018-2) algunos de ellos cursaban de sexto a octavo semestre, otros habían egresado, se encontraban ya titulados, habían ingresado al posgrado o se encontraban laborando. Son 10 mujeres y 10 hombres, con edades de 20 a 30 años en el momento del estudio. Los datos más relevantes se representan en la tabla 1. Se indica el campo de conocimiento principal que cursaron o están cursando, así como si lo combinan con otros campos o han tomado por lo menos alguna asignatura que no sea de su campo principal.

Se invitó a los estudiantes de manera presencial y por red social a participar en el estudio; se integraron los 20 casos que accedieron a participar voluntariamente en la entrevista y a compartir su historia académica. Se acordó con ellos el manejo confidencial de su nombre y el uso de su información sólo con fines e investigación. Asimismo, se realizó el proceso de devolución de la información para la validación del análisis y conclusiones.

Tabla 1. Participantes del estudio

\begin{tabular}{|c|c|c|c|c|c|c|c|}
\hline Caso & Género & Edad & Generación & Semestre & Promedio & Campo principal & Otros campos \\
\hline E1 & M & 20 & 2016 & $6^{\circ}$ & 8.88 & Educativa & $\begin{array}{c}\text { Clínica, Social y Ciencias Cognitivas } \\
\text { y del Comportamiento (ccc) }\end{array}$ \\
\hline E2 & M & 22 & 2014 & $\begin{array}{c}8^{\circ} \\
\text { Tesis SS }\end{array}$ & 9.50 & Educativa & Clínica \\
\hline E3 & $\mathrm{H}$ & 20 & 2015 & $6^{\circ}$ & $9.45^{*}$ & Educativa & $\begin{array}{l}\text { No } \\
\text { Estudia Música }\end{array}$ \\
\hline E4 & $\mathrm{H}$ & 21 & 2016 & $6^{\circ}$ & 9.40 & Educativa & No \\
\hline E5 & M & 23 & 2013 & $\begin{array}{l}\text { Egresada } \\
\text { sS }\end{array}$ & $9.01^{*}$ & Educativa & Social \\
\hline E6 & M & 21 & 2015 & $6^{\circ}$ & 9.71 & Educativa & No \\
\hline E7 & M & 21 & 2015 & $6^{\circ}$ & 9.62 & Social & Educativa \\
\hline E8 & $\mathrm{H}$ & 22 & 2014 & $\begin{array}{c}8^{\circ} \\
\text { Tesis SS }\end{array}$ & 9.14 & Educativa & No \\
\hline
\end{tabular}




\begin{tabular}{|c|c|c|c|c|c|c|c|}
\hline Caso & Género & Edad & Generación & Semestre & Promedio & Campo principal & Otros campos \\
\hline E9 & M & 24 & 2014 & $6^{\circ}$ & $8.28^{*}$ & Educativa & Clínica \\
\hline E10 & $\mathrm{H}$ & 21 & 2015 & $6^{\circ}$ & 9.37 & Clínica & Educativa \\
\hline E11 & $\mathrm{H}$ & 26 & 2012 & $\begin{array}{c}\text { Licenciado } \\
\text { Estudia Maestría }\end{array}$ & 9.23 & Educativa & Posgrado en Pedagogía \\
\hline E12 & $\mathrm{H}$ & 24 & 2012 & Licenciado & 9.31 & Educativa & Social \\
\hline E13 & M & 23 & 2015 & $8^{\circ}$ & 9.80 & Neurociencias & ccc Social \\
\hline E14 & $\mathrm{H}$ & 25 & 2012 & $\begin{array}{l}\text { Licenciado } \\
\text { Trabaja }\end{array}$ & 9.10 & Educativa & Clínica Organizacional \\
\hline E15 & $\mathrm{H}$ & 22 & 2015 & $\begin{array}{l}\text { Egresado } \\
\text { Tesis }\end{array}$ & 9.66 & Social & Educativa Clínica \\
\hline E16 & $\mathrm{H}$ & 24 & 2013 & $\begin{array}{c}\text { Licenciado } \\
\text { Estudia Maestría }\end{array}$ & 9.36 & Educativa & Social Posgrado MADEMS \\
\hline E17 & $\mathrm{H}$ & 30 & 2013 & $\begin{array}{l}\text { Egresado } \\
\text { Tesis Trabaja }\end{array}$ & 8.88 & Social & Educativa \\
\hline E18 & M & 23 & 2014 & $\begin{array}{l}8^{\circ} \\
\text { Tesis SS }\end{array}$ & 8.90 & Neurociencias & $\mathrm{CCC}$ \\
\hline E19 & $M$ & 21 & 2014 & $\begin{array}{l}8^{\circ} \\
\text { Tesis ss }\end{array}$ & 9.71 & Educativa & $\mathrm{ccc}$ \\
\hline E20 & M & 28 & 2010 & $\begin{array}{l}\text { Egresada } \\
\text { Tesis Trabaja }\end{array}$ & 9.40 & Clínica & Educativa \\
\hline
\end{tabular}

$\left(^{*}\right)$ Han reprobado o no presentado alguna asignatura en la licenciatura. Se indica cuando cursan el servicio social (ss) o están realizando tesis.

\section{Instrumento y procedimiento}

Se conformó una guía de 14 preguntas de respuesta abierta sobre el proceso de elección de trayectorias académicas personales de aprendizaje, la cual se aplicó de manera personal, ya sea en el formato de entrevista audiograbada o de cuestionario escrito, en función de las facilidades brindadas por el participante. La guía se elaboró y validó con dos académicas expertas en currículo y formación profesional para ajustar su contenido, el cual se derivó de las preguntas de investigación. Todos los participantes compartieron a los investigadores copia de su historia académica y tira de materias más reciente, la cual fue comentada con ellos en la entrevista. Cuando se consideró necesario (tres casos) se solicitó una entrevista adicional para profundizar en algunos aspectos. Los datos se codificaron en plantilla Excel y se procedió a su análisis cualitativo.

\section{Resultados}

Con base en el análisis de contenido y la discusión con los estudiantes del sentido que otorgan a su TPA y cómo conciben la flexibilidad curricular, se identificaron algunas tendencias que se exponen a continuación y se ofrecen diversos extractos discursivos que ilustran sus argumentos.

\section{Cómo conforman los estudiantes sus trayectorias personales de aprendizaje}

En todos los casos se puddieron identificar los 
motivos y argumentos de los participantes en su proceso de elección de asignaturas optativas en el tramo de formación profesional; es decir, la conformación de la TPA tiene una lógica determinada y se basa en la información disponible por el estudiante, quien busca dar respuesta a su propia mirada en torno a la disciplina y la profesión.

Así, se encontró que la elección está basada principalmente en los intereses y preferencias personales de los estudiantes respecto a los distintos enfoques y campos teóricos y profesionales de la psicología; en segundo término, está en función de alguna experiencia formativa concreta o algún objeto de estudio que se asume como propio; y sólo en algunos casos se basa en información sobre el campo laboral. En la elección de campo de conocimiento un aspecto esencial reside en la influencia de los profesores con los que han cursado asignaturas en los primeros semestres, en el área de formación general.

El momento en que se toman las decisiones procedentes y se busca la información requerida para ello, ocurre en cuarto semestre; sólo una estudiante reportó prever su TPA desde tercer semestre. Muchos de los estudiantes entraron a la carrera con la idea de cursar determinada área profesional de la psicología, prioritariamente el campo de la clínica; algunos mantuvieron dicha previsión, pero otros la cambiaron durante los cuatro primeros semestres. A continuación de plasman diversos extractos discursivos que dan cuenta de las perspectivas de los y las jóvenes en formación. Nótese la diversidad de intereses, objetos de estudio y escenarios de la profesión que les interesan, así como la influencia de las experiencias que van logrando en la carrera.

[...] me interesa el fenómeno de la educación, mi atención fue captada por este campo debido a la teoría sociocultural de Vigotsky [...] quiero convertirme en un gran investigador (E4).

Pertenezco mayormente al área educativa debido a mi interés por la calidad en la educación [...] combiné con el área social porque considero que es importante tener un panorama más amplio sobre la realidad social y los fenómenos que en ella ocurren (E5).

Social lo elegí, en un principio, por la psicología social comunitaria, ya que era uno de los campos en los que más me interesaba incursionar [...] En educativa me interesé también por la cuestión del ámbito laboral, ya que viéndolo a largo plazo me pareció más conveniente poder tener herramientas de un área donde, entre comillas, tal vez podría resultarme más sencillo conseguir un empleo (E7).

Me pareció importante combinar educativa con social debido a que ambas ofrecían prácticas en escenarios externos y en temas de mi interés que me ayudarían a desarrollar competencias importantes para comprender y mejorar algunos proyectos sociales en los que estaba participando en colaboración con algunos compañeros externos a la universidad, de la Facultad de Psicología y de la Facultad de Filosofia (E17).

[...] la psicología clínica es la base para comprender el comportamiento humano y ahí pude desarrollar diversas competencias y habilidades que ahora utilizo en el campo de la educación donde trabajo actualmente (entrevista, rapport, elaboración de informes, observación, etcétera) (E20).

\section{Criterios para la toma de decisiones respecto a la TPA}

Los estudiantes son conscientes de la importancia de esta toma de decisiones respecto a su TPA y lo asumen como proyecto de carrera e incluso de profesión y vida. Entienden la responsabilidad social que implica dicha elección. Reportaron que es esencial comprender que la licenciatura contempla dos grandes momentos. En principio, la formación general, que opera como común denominador a todos y que ubican como esencialmente "teórica", con cursos obligatorios, basados en la exposición magisterial y un segundo tramo, la formación profesional, donde 
eligen al menos un campo profesional de interés y lo pueden combinar o complementar con asignaturas de otros campos. En esta segunda etapa, que les genera una mayor motivación, la expectativa es tener una formación mucho más "práctica", "aplicada" o "profesional".

En su mayoría, expresan una visión y expectativa de la psicología en una lógica de diversidad, no de unicidad, dado lo que han aprendido en torno a las distintas tradiciones o paradigmas psicológicos. Esto es un reflejo de su concepción de la psicología misma, pluriparadigmática, aunque reconocen no lograr integrar una visión de conjunto de la disciplina. Los estudiantes mencionan que tienen definidos objetos de estudio acotados y trayectorias profesionales o de investigación a las que se quieren dedicar y eso preside sus elecciones en la formación profesional (por ejemplo, formarse en evaluación psicológica, en educación infantil, en psicología comunitaria, como investigador en procesos de cognición, como especialista en inclusión de personas con discapacidad, como experto en medicina conductual, entre otros). Como disyuntiva, algunos plantearon que salen como psicólogos generales, pero saben que el campo de trabajo los llevará a especializarse. Sólo una alumna se representó la profesión con un carácter general, aunque acotado a una línea de intervención de su interés (el desarrollo infantil, respecto al que dijo querer saber algo desde la clínica, la educación, las neurociencias).

Cabe mencionar que algunos estudiantes perciben disputas entre diferentes paradigmas y grupos afiliados a teorías y tradiciones psicológicas, mencionan grupos de poder y dicen que algunos docentes "les hablan mal" de otros departamentos, lo que genera confusión cuando se eligen trayectorias:

[... que se dé a conocer lo que se hace desde cada psicología, y no solo que la Facultad eche la casa por la ventana por una o dos versiones de la psicología [..] fortalecer y sistematizar el programa de tutoría, sea por la beca o no, tener un tutor crítico, imparcial y libre de proselitismo académico debería ser una estrategia principal en la Facultad. Así que, definitivamente, deben fortalecer al estudiantado para que construya su trayecto (E11).

En el discurso de todos los participantes se mencionan como criterios de elección principal de las asignaturas a cursar dos aspectos: el contenido del programa y el docente a cargo del grupo. Con base en esto conforman su plan de TPA semestral, el cual se puede modificar en función de los horarios y los mecanismos de inscripción, que operan como los principales problemas para seguir la trayectoria prevista. Sólo en algunos casos toman en cuenta el campo laboral (lo cual ocurre hasta los últimos semestres) y hay estudiantes que arman su TPA en función de lo que requieren para continuar en un posgrado.

[¿̇Tomó en cuenta el campo laboral?] Muy poco, consideré mucho más las materias que requería para seguir una trayectoria en Posgrado (E6).

No me informé de los campos de trabajo del psicólogo de la educación ni de las competencias que se requieren en los escenarios reales (E14).

Fue hasta los últimos semestres en los que me preocupé por el campo laboral y me puse a investigar la oferta de trabajo y salarios sobre mi área en la que me estoy formando (E19).

\section{Fuentes de información para decidir una TPA}

La estrategia más reportada para la toma de decisiones respecto a cómo conformar la propia TPA es de búsqueda independiente; todos reportaron que en su momento buscaron por cuenta propia información sobre las asignaturas del campo de conocimiento elegido en el sitio web de la Facultad de Psicología y consultaron la oferta académica en los horarios del periodo a cursar. Particularmente, se enfocan a comprender la lógica de acumulación de créditos y 
cuando es de su interés, de combinación posible de asignaturas de distintos campos de conocimiento. Reportan realizar elecciones informadas y muchas veces basadas en el plan de carrera que han construido previamente; aunque las opiniones de otros integrantes de la comunidad, sobre todo de los pares, son tomadas en cuenta.

La siguiente explicación parece coincidir con lo que hacen muchos de los estudiantes para conformar su trayectoria, e implica el abordaje estratégico mediante el cual deciden su TPA en lo concerniente al tránsito a la formación profesional en licenciatura:

Me daba tiempo para elaborar hasta más de tres propuestas de horarios previendo incompatibilidad de horario y falta de cupo. Las propuestas de horarios giraban en torno a una o dos materias que consideraba eran las más interesantes por su contenido y por los/las profesores/as que las impartían, recuerdo que la materia Contextual me parecía la menos importante por lo que era la última en ser acomodada en las propuestas de horarios. La fuente principal fueron los documentos contenidos en la página de la Facultad. Cada semestre revisaba el plan de estudios y la ficha descriptiva de cada materia que me interesaba para ver el contenido del curso. Luego dedicaba un tiempo a buscar información acerca de los profesores usando Google. Esto me ayudaba a encontrar currículums profesionales de profesores, videos de conferencias, temas de investigación y hasta comentarios hechos por compañeros en la página de misprofesores.com y grupos de Facebook. Sinceramente, en muchos casos la información era insuficiente para una decisión definitiva, pero elemental al momento de descartar horarios en la etapa de "Altas y Bajas", fase muy importante en cada semestre para establecer la tira de materias y horarios definitivos (E17).

Como se observó en la tabla 1, la mayoría (16) de los participantes combinaron su campo de conocimiento principal con otros contenidos, mientras que sólo cuatro jóvenes se pronunciaron por querer estudiar exclusivamente el campo elegido. Llama la atención el tipo de combinaciones elegidas: educativa-social y neurociencias-CCC, mientras que clínica suele combinarse con prácticamente todos los demás campos, sobre todo porque los alumnos consideran que hay competencias básicas en dicho campo que son nucleares en la formación del psicólogo, y que debe poseer todo profesional (entrevista, psicodiagnóstico, construcción de pruebas psicológicas). Para la mayoría, la psicología clínica sigue definiendo la profesión ante la sociedad. Por su parte, los estudiantes que cursan asignaturas en psicología organizacional dicen que es el área que se percibe con mayor oferta laboral y consideran inadecuado que no sea estudiada desde la formación general básica.

Esta información se trianguló con las historias académicas proporcionadas por los estudiantes, donde en efecto aparecieron dichas combinaciones entre campos de conocimiento como las más frecuentes. No obstante, la lógica y sentido de la trayectoria sólo es posible entenderla desde la argumentación de los intereses y objetos de estudio a los que han arribado los estudiantes, quienes afirman que, al pasar a la formación profesional, dado que están cursando lo que les interesa, se sienten mucho más motivados y se incrementa su rendimiento académico, por lo que en general reportan que su promedio sube hacia el final de la carrera. Para muchos estudiantes, lo que genera mayor motivación y compromiso es la asistencia a escenarios reales, y comentaron que es el principal déficit del plan de estudios desde la formación general, el que no haya suficiente formación en la práctica de la profesión.

Algunos estudiantes mencionaron que en la asignatura de $4^{\circ}$ semestre Comprensión de la realidad social 1, hay docentes (aunque no todos) que les informan puntualmente cómo planear sus trayectorias y les hacen profundizar en el conocimiento del o los 
campos profesionales de su interés para que tomen decisiones informadas. En mucho menor medida se reporta buscar el apoyo o interlocución con las autoridades (coordinadores o directivos), y ocasionalmente toman en cuenta las actividades (pláticas informativas, conferencias, etcétera) que organizan las coordinaciones académico-administrativas; es decir, la mejor recomendación (y credibilidad) proviene de sus pares y de docentes en los que confían.

Algunos reportan haber recibido el apoyo de tutores. Sin embargo, la experiencia de la tutoría es valorada favorablemente por quienes la han tenido, mientras que los que no han tenido tutoría consideran que sólo es necesaria cuando se presentan determinadas problemáticas. Hubo estudiantes que dijeron haber elegido por su cuenta un tutor "no oficial", un académico con quien se identifican y del que han tenido apoyo personal y académico, que puede ser el docente de algún curso, de sus prácticas en escenarios o el director de tesis o servicio social. Uno de los jóvenes comentó que debería instaurarse un modelo de tutoría entre pares.

No, no cuento con ninguno, pero tampoco lo considero tan importante (E2).

Sí cuento con un tutor, pero no formalizado. A quien llamo "mi papá académico" (E3).

Fue mi tutora (esto por el programa de la beca Manutención, antes PRONABES) desde tercer semestre, quien me guió, asesoró y acompañó durante toda mi formación, algunos de los ejercicios que me solicitó fue hacer un plan de vida y carrera (E11).

Sería interesante que se abrieran programas de tutorías en los que se le dé la oportunidad a jóvenes de semestres avanzados o egresados de la Facultad de ser los tutores (E16).

\section{¿Trayectorias indicativas y flexibles o preestablecidas y obligatorias?}

La totalidad de participantes de este estudio se pronunció a favor de que el estudiante elija libremente su propia trayectoria personal (campo de conocimiento y asignaturas) en el tramo donde definen a qué se quieren dedicar en la profesión. Afirman que llegado ese momento los estudiantes son adultos competentes para la toma de decisiones sobre lo que quieren cursar a condición de estar informados. Mencionan como un componente favorable del currículo la flexibilidad de éste y la amplitud de opciones para la formación. Se manifestaron una serie de inconvenientes en el caso de que las asignaturas del tramo de formación profesional fueran obligatorias, principalmente, el impedir una TPA ajustada al plan de carrera e incluso de vida del futuro profesional de la psicología.

Considero que cada estudiante es capaz y puede elegir de manera libre las materias a elegir, el campo de la psicología es muy amplio y diverso y creo que sería un error que la trayectoria académica de todos fuera la misma porque siendo de esta manera cerraríamos y reduciríamos la visión y lo rico de la disciplina (E2).

[elección libre] Ya que "a estas alturas del partido" lo ideal es que cada estudiante visualice lo que quiere hacer en la psicología y en la vida. El poder escoger mis materias me ofrece una sensación de control y agencia sobre mi futuro y trayectoria. El que se me obligara a tomar materias obligatorias me parecería una imposición y ofrecería resistencia ante ello (E3).

[...] la característica de flexible dentro del plan de estudios es un buen añadido, que nos permite acercarnos, especializarnos y hacernos expertos en la segunda mitad de la carrera para así tener mejores oportunidades no solo de entrar a un posgrado sino también de poder desempeñarnos mejor en el campo laboral o poder competir internacionalmente (E4).

Sí, deben elegir libremente. Porque, en primer lugar, responde a los intereses de los compañeros. Por otro lado, al ser la psicología una disciplina que se fundamenta en la convergencia de otras disciplinas 
y que se nutre de la colaboración con un sinfín de profesionales, me parece que elegir la propia trayectoria impulsa a la formación de psicólogos diversos para una realidad compleja e interconectada, y a establecer la importancia de las contribuciones de todos los campos de conocimiento, rompiendo con figuras autoritarias de la ciencia del siglo XX y a la educación bancaria y en masas, donde se forman fotocopias de profesionales (E12).

Considero que sería importante que desde primer semestre se den materias a elegir, quizá en menor cantidad para que siga existiendo un tronco común, pero también se podría dar la opción de trayectoria sugerida para personas que no tengan un plan en mente, quizá a partir de tutorías iniciales o algún software que tome en cuenta intereses. Pero la elección libre de materias y profesores debería ser fundamental para cubrir la diversidad de intereses que hay en la comunidad estudiantil (E17).

[...] hay campos del conocimiento que pueden complementarse y los intereses de la gente pueden y van cambiando conforme nos exponemos a profesores, materias e información (E18).

Aunque se inclinan por una pre-especialización profesional en el último tramo de la licenciatura (así denominado en la normatividad universitaria y que en este plan de estudios se define como campo de conocimiento), reconocen que hay contenidos que deberían ser comunes para todo psicólogo y que no están debidamente atendidos en la formación profesional o que deberían darse desde los semestres básicos con carácter obligatorio (entrevista, psicodiagnóstico, pruebas psicológicas, estadística, métodos cualitativos, entre los más mencionados).

Para algunos estudiantes, a quienes se debe imponer lo obligatorio es a los profesores, porque en muchas ocasiones no imparten el programa del curso y con ello afectan la trayectoria prevista por el estudiante. En otros casos, afirman que los contenidos están sesgados a una sola mirada disciplinar, por lo general experimentalista o positivista, omitiendo otras que consideran relevantes, situación que puede ubicarse como parte del currículo "nulo" y no se imparte, o bien que es relevante y está ausente en los programas.

Sugeriría programas de fortalecimiento a la práctica docente y mayor "control" porque muchos [profesores] aplican mal la libre cátedra y esto afecta a la formación de los alumnos (E2).

En el campo de Ciencias Cognitivas y del Comportamiento, se requiere dar cabida a más espacio para las ciencias cognitivas, las asignaturas prevalentemente siguen girando en torno al conductismo y las pocas materias que se relacionan con cognición sólo muestran el paradigma computacional clásico cuando existen varias perspectivas teórico-metodológicas (E13).

En el campo laboral solicitan psicólogos con conocimientos y habilidades específicos de alguna área [...] considero que no está bien que se forman psicólogos que tratan de ser "todólogos" (E19).

Un estudiante, que ya es egresado, está titulado y se encuentra laborando, considera que lo que debería ser obligatorio es llevar cursos fuera de la Facultad de Psicología; no obstante, carece de información respecto a que la movilidad estudiantil es un hecho consignado en la legislación universitaria y que podría cursar hasta un 30-40\% de sus estudios en otra entidad o universidad y también tiene la opción de cursar asignaturas equivalentes de su interés en otra escuela o facultad:

Creo indispensable la formación transdisciplinaria, por lo que sugeriría que volvieran obligatorio el tener que elegir cursos fuera de la Facultad de Psicología, de manera que su formación resulte complementada y se pueda realizar un análisis más completo de los límites, ventajas y desventajas de la disciplina psicológica (E14). 


\section{Aspectos que favorecen y obstaculizan la TPA}

Los principales obstáculos que encuentran los estudiantes para llevar a cabo su TPA como la han planeado se ubican en el plano de la gestión administrativa y la planeación semestral de la oferta académica: mecanismos de inscripción, horarios, docentes que imparten las asignaturas y programas que no se cumplen. Algunos indican que sigue habiendo problemas con el sistema informático por sobresaturación de la red al momento de la inscripción. También mencionaron que quitaron un simulador informático de trayectoria digital y diversos videos informativos, así como folletos que se bajaban de la red para los estudiantes.

Conforme a su experiencia, los participantes se dividen en los que sí lograron inscribirse en los cursos y con los profesores que deseaban, aunque en este caso, eso los obligó a tener horario mixto, con muchas horas de desfase entre las clases que tomaban al día. Los estudiantes que mencionan que se han visto obligados a tomar cursos que no forman parte de sus intereses o no encajan en su proyecto de formación personal, lo atribuyen a problemas con los mecanismos de inscripción y horarios, por cuestiones de los créditos que tienen que completar o bien porque se han visto forzados a inscribirse con profesores que no deseaban dado el cupo límite de los cursos.

Siempre ha sido un problema lograr encontrar un horario que me guste y desde segundo semestre mi horario ha sido mixto y siempre he tenido una materia en la que no estoy ni con el profesor que quiero, ni en el horario y es una materia a la que me da mucha pereza asistir (E1).

La página de la Facultad al momento de la inscripción siempre resulta ser un factor de estrés ya que se satura y, por tanto, cuando carga ya no hay lugares en varios grupos (E8).

Al llegar a octavo semestre, me faltaría 1 crédito, ¡sí, sólo 1! Es algo bastante raro, detalle que quizá este claustro (Educativa) debió cuidar, ya que debí tomar una materia de 6 créditos para "pagar" 1 [...] el crédito huérfano o fantasma [...]; esa clase me desmotivó ya que fue una especie de relleno y era la que sin duda exigía desproporcionalmente más que las otras que sí había metido por gusto y para concretar mis intereses (E11).

Tuve la fortuna de tener todas las asignaturas que tenía contempladas, por lo tanto, no hubo alguna asignatura que no me fuera útil (E16).

Llegué al punto de buscar y establecer personalmente acuerdos con algunos profesores para que me pasaran calificación a pesar de no estar inscrito. Podría decir que esa opción "me salvó el semestre" en varias ocasiones pues era pesado ir a clases con profesores que no deseaba o todavía más pesado llevar materias de relleno para cubrir créditos (E17).

La problemática respecto a los profesores con los que no quieren cursar materias la ubican en su falta de cumplimiento del programa, su desinterés por el estudiantado, la mala relación con el mismo, su actitud o autoritarismo, la inasistencia docente a las clases.

[...] era terrible que tres materias te muestren algo en lo que realmente no estás interesado y que no cumplan las expectativas del programa. Me decepcioné bastante, pero aun así aprendí (E5).

$[\ldots]$ no me gustaba la forma en la que los docentes impartían la clase. Tenían una perspectiva muy rígida, jerárquica e impositiva dentro del salón de clases lo cual me hacía sentir incómoda y sin ganas de participar, pues reprimían bastante la crítica en general (E7).

$\mathrm{Al}$ no contar con bases sólidas que otorgan gran parte de los profesores en tronco común, en ocasiones llegaba a pensar en cambiarme a FES Zaragoza por los atributos que se tienen del área clínica y de la salud (E10).

Fue muy complicado comprenderle al profesor y esto se convirtió en un problema en común en el 
grupo al punto de que la cantidad de asistencia de alumnos a las clases disminuyó a menos de la mitad para el primer mes. Los temas eran buenos, pero no pude rescatar mucho de ellos debido a la forma en que se abordaron, poco clara, acompañada de una negativa relación docente-alumnos (E17).

No obstante, también se reportan experiencias que los estudiantes consideran muy favorables para su formación como psicólogos y como personas gracias a la interacción con algunos de sus docentes:

Con la doctora $x$ tuve una mirada fresca respecto del área de educativa y los procesos de aprendizaje, pude vislumbrar completamente lo que significa ser creativa al momento de impartir una clase y sirvió muchísimo para engancharme al área (E7).

La maestra $x$ fue excelente en proporcionarme el conocimiento completo para la aplicación e interpretación de las pruebas psicológicas (E10).

Para algunos estudiantes, un problema es la forma en que autoridades y docentes perciben al alumnado de psicología, como estudiantes no competentes para tomar sus decisiones, por lo que los ubican ajenos a lo concerniente al currículo:

Que se deje de pensar que los alumnos somos ignorantes y no sabemos qué queremos (E4).

Se necesita que todos los estudiantes participen dando su opinión para la creación de planes de estudio y no sea una decisión impuesta por autoridades, que sólo conocen la psicología desde su campo del conocimiento (E18).

En el caso de los tres estudiantes que habían reprobado uno o más cursos, se indagó esta situación y se evidenció que ser alumno regular o no regular puede ser algo transitorio. En este sentido, los alumnos plantearon que, al cambiar el carácter de los cursos de la licenciatura a obligatorios en su mayoría, podría haber más rezago académico entre los estudiantes, perjudicando su avance en la licenciatura e incrementando no sólo la desmotivación, sino los recursamientos y exámenes extraordinarios. Los motivos por los que no aprobaron determinados cursos, los asocian a desinterés por la materia o la forma de impartirla, actitudes del docente hacia el grupo, problemas con el horario, enfermedad. Nótese que son estudiantes con buen promedio académico y a pesar de ello tuvieron experiencia de reprobación y rezago.

Algunos estudiantes mencionaron que la carga extraclase de algunas asignaturas, y particularmente en algunos campos de conocimiento, es excesiva. El que los programas no indiquen el tiempo extra que habrán de dedicar a la asignatura más allá del aula para dar cumplimiento a las actividades de aprendizaje, realizar trabajos, preparar exámenes, etcétera. incrementa significativamente su carga académica sobre todo al final del semestre; dejan de atender las demás materias del semestre por estudiar para un examen específico, y eso ha sucedido desde semestres básicos, sobre todo en Neurociencias. En otros casos, al arribar a los últimos semestres la carga académica se hace muy intensa debido a que se comienza a cubrir el servicio social o a realizar la tesis, y algunos estudiantes participan en proyectos de investigación e intervención, otros comienzan a trabajar:

En esta asignatura [Neurociencias] tuve una carga de trabajo desproporcionada en comparación con el número de créditos que se le otorga, además la persona a cargo de la asignatura carecía de los conocimientos necesarios [...] faltó en repetidas ocasiones $[\ldots]$ en el trabajo final no se brindó ningún tipo de asesoría (E13).

En ese entonces $\left(8^{\circ}\right.$ semestre $)$ estaba haciendo servicio social, prácticas profesionales, trabajo de tesis, trabajo de investigación como becario y trabajo remunerado, me dividí en muchos yo's para poder cumplimentar con todo ello, creo que es un elemento que, al menos Educativa debe velar (E11). 
$\mathrm{Al}$ revisar las historias académicas, en todos los casos se observa que los estudiantes eligen cursos tanto de formación en contenidos teóricos, como metodológicos y de práctica en laboratorios o escenarios fuera de la universidad. Incluso, algunos reportan haber realizado prácticas en cursos que están catalogados como teóricos, sobre todo en campos como clínica, educativa y social. Sólo se presentó el caso de dos estudiantes que reportaron no haber realizado prácticas en escenarios profesionales. Los demás (18) afirmaron que dicha formación es de lo más valioso en su trayectoria. En general, los estudiantes mencionaron que se necesita de una docencia innovadora, de la apropiación de tecnologías del siglo XXI y de mayor acercamiento a la realidad de la profesión, no sólo a las teorías psicológicas.

[Las materias teórico-prácticas, en escenarios] Te hacen valorar lo importante, tener una visión crítica, ver los intereses, a las personas involucradas. Simplemente, te hace ver que todo es muy diferente a como lo ves en un salón de clase y que lo importante no son los números, no es un "sujeto" vacío sin nombre flotando en la nada. Son personas en un contexto especifico, con necesidades, creencias, costumbres, valores, tradiciones, cultura, estructura social, únicas y diferentes [...] A ver lo complejo del fenómeno, a tener esta mirada humanista decolonial [...] Y he de decir que por ello decidí ser investigador (E4).

[La formación en escenarios] me ha servido para pulir radicalmente mis habilidades en manejo de grupos, en fuentes de información, herramientas emocionales, habilidades discursivas y también organizativas, pues el trabajo que llevamos a cabo lo hacemos constantemente en equipo y nos vinculamos con otras áreas de la psicología y con otros profesionistas que puedan aportar al proyecto (E7).

Dichas asignaturas me permitieron darme cuenta de que es posible realizar intervenciones psicosociales desde una visión crítica y emancipadora. En donde no se ve a las personas como individuos aislados o personas enfermas, sino como comunidades vulneradas que tienen las herramientas y el conocimiento para transformar su misma realidad. A la par, las prácticas me permitieron poner en práctica el conocimiento metodológico y teórico que ya tenía y me permitió desarrollar nuevos. Aunque lo más importante fue la formación ética y política que obtuve (E15).

\section{Lo que los estudiantes requieren en su formación como psicólogos}

Los participantes ofrecieron una diversidad de propuestas para la mejora de la formación que reciben en la licenciatura, algunas de las cuales se reproducen a continuación:

Que [el plan de estudios] siga siendo flexible, que se deje de centrar tanto en contenidos y en lugar de eso se centre en desarrollar un razonamiento científico en psicología. Que se vea que la psicología es más que una rata, un laboratorio, un reforzador y una tabla con estadística, que lo importante son las personas, la desigualdad, las enfermedades, la segregación y que de esta manera veamos que es importante formarnos de mejor manera [...] se deje de pensar que los alumnos somos ignorantes y no sabemos qué queremos y se respete y fomente la flexibilidad de currículum, pero sobre todo confien en que nosotros los estudiantes y jóvenes somos lo suficientemente responsables como para saber elegir sobre nuestra preparación (E4).

Evaluar el currículo oculto y las prácticas de enseñanza-aprendizaje que se llevan a cabo en las aulas de la Facultad. Porque el planteamiento u organización curricular parece no corresponder con la realidad de muchos compañeros, ocasionando preguntas de si el error está en el plan o en la falta de evaluación y seguimiento de lo que ocurre dentro de las aulas (E6).

Sería conveniente que las y los estudiantes de la carrera pudiesen hacer prácticas o estar en contacto 
en escenarios reales desde semestres anteriores al quinto (E10).

Los conocimientos para el emprendimiento de proyectos propios. Se nos menciona que el campo laboral es muy competitivo y escaso, pero no parece haber una propuesta en cuanto a que los propios psicólogos intervengan desde sus propias fortalezas con un proyecto propio, socialmente relevante y responsable (E12).

Específicamente para el caso del área de Psicobiología y Neurociencias, se requiere un abordaje más claro del papel del psicólogo dentro de este campo. Por otra parte, existen vacíos que requieren tratarse dentro de la formación académica de los estudiantes, puesto que se da por sentado el conocimiento de biología molecular, estadística y bioestadística, etología, evolución, etcétera, cuando dentro del plan de estudios no se da cabida a una formación sólida en éstas, siendo que queda en los estudiantes cubrir estos vacíos (E13).

Deberían existir asignaturas en donde se vea contenido político. Greo que algo que no nos caracteriza como facultad es el conocer la agencia política que tiene la psicología dentro de la modernidad y nuestra sociedad [...] Sensibilizar a los alumnos y alumnas para que nos percatemos de esto me parece fundamental, y que sin duda está completamente ausente en nuestro plan de estudios actual (E15).

\section{Conclusiones}

Desde su propia perspectiva, los estudiantes de licenciatura quieren, saben y pueden elegir su TPA y prefieren hacerlo con plena libertad entre una amplia oferta de asignaturas optativas, reconociendo que debe ser una elección informada. Su interés reside en conformar trayectos tan personalizados como sea posible, lo que coincide con las actuales tendencias curriculares y educativas (Coll, 2016; Loveless y Williamson, 2017). Esto se ajusta a un proyecto de formación propio, basado en sus intereses, objetos de estudio o ámbitos donde desean intervenir como profesionales de la psicología.

Los estudiantes explicaron el proceso mediante el cual conforman sus TPA, con base en una búsqueda de información sobre contenidos de las asignaturas del o los campos de conocimiento elegidos, principalmente en el sitio web de Facultad. Asimismo, buscan y comparten información respecto a los docentes que las imparten, siendo éste un factor decisivo y que define para ellos lo provechoso o no del curso. Consideran que las principales restricciones se vinculan con la gestión del currículo, y residen en el número de créditos a cubrir, en la oferta de horarios existente y en los mecanismos de inscripción.

Una problemática mencionada con frecuencia es que los docentes no imparten el programa oficial de la asignatura o lo hacen de manera tal que no se propicia la motivación ni el aprendizaje. Entre las principales deficiencias en la formación que los estudiantes proponen que se atiendan en la formación básica común a todo psicólogo, mencionan entrevista psicológica, psicodiagnóstico y construcción de instrumentos, métodos de análisis cuali y cuantitativos, pensamiento crítico y político, así como una formación práctica en escenarios reales desde el inicio de la licenciatura. Reconocen que el tema de las tecnologías digitales de punta está ausente de su formación en y para la profesión. Lo expresado por los participantes refrenda la importancia de que la institución disponga de diversos recursos, materiales y mecanismos de apoyo a la conformación de las trayectorias estudiantiles, entre los que se encuentra el fortalecer mecanismos de información y tutoría para la elección de TPA. Los estudiantes aportaron una diversidad de propuestas para la mejora de su formación como futuros profesionales de la psicología y enfatizaron la necesidad de una buena formación y evaluación de los docentes, acorde a la diversidad del currículo a lo que la profesión demanda en la actualidad.

Finalmente, encontramos que los estudiantes universitarios son personas dotadas de agencialidad y 
capacidad para la toma de decisiones respecto a su trayectoria, que logran tomar decisiones informadas ajustadas a sus intereses y valoran la flexibilidad del currículo en la medida que les permite conformar una trayectoria personalizada de aprendizaje de la profesión. Como parte de su formación han logrado vislumbrar un panorama amplio de la psicología como profesión, aunque insisten en que no logran el nivel de competencia deseado en ámbitos que pueden identificarse como el currículo nulo de la carrera: lo que se omite o minimiza, pero que es importante desde la perspectiva de los actores del currículo.

\section{Referencias}

Barron, B. (2010), "Conceptualizing and tracing learning pathways over time and setting", NSSE Yearbook, National Society for the Study of Education, núm. 1, pp. 113-127.

Barrón, C. y F. Díaz Barriga (2016), "Curriculum management and the role of curriculum actors", Transnational Curriculum Inquiry (TCI), vol. 13, núm. 2, recuperado de <http://ojs.library.ubc.ca/index.php/ tci/article/view/188285/186427> [Consulta: marzo de 2019].

Coll, César (2016), Personalización del aprendizaje escolar, México, Fundación SM.

Díaz Barriga, F., F. Soto y A. Díaz (2015), "Los estudiantes universitarios como actores del currículo: reflexión de su trayecto a través de relatos digitales personales", Currículo sem Fronteiras, núm. 3, pp. 626-644.

Díaz Barriga, F., M. M. Pérez y Y. Lara (2016), "Para enseñar ética profesional no basta con una asignatura: los estudiantes universitarios de Psicología reportan incidentes críticos en aulas y escenarios reales", Revista Iberoamericana de Educación Superior (RIES), vol. VII, núm. 18, pp. 42-58.

Díaz-Villa, Mario (2008), "Lectura crítica de la flexibilidad", La educación superior frente al reto de la flexibilidad, Bogotá, Editorial Magisterio, pp. 177-205.
El discurso de los jóvenes nos permite corroborar lo que han encontrado Guzmán y Saucedo (2007: 12) en sus estudios sobre estudiantes universitarios: son sujetos activos, que construyen día a día su identidad y buscan continuamente el sentido de su quehacer. Finalmente, son jóvenes adultos que no pueden ni quieren concebir el currículo de la profesión que estudian como un camino cerrado o único prescrito por los expertos o las figuras de autoridad, que no están dispuestos a convertirse en "fotocopias de profesionales" y que quieren que su voz sea tomada en cuenta.
Escalona, L. (2008), "Flexibilidad curricular", Investigación Bibliotecológica, núm. 44, pp. 143-160.

Facultad de Psicología (2008), Plan de estudios de la Licenciatura en Psicología, México, Facultad de Psicología (Sitio web oficial de la Facultad de Psicología), UNAM, <http://www.psicologia.unam.mx/documentos/ pdf/Licenciatura_Psicologia_UNAM_Plan_de_ Estudios_2008_Informacion_y_estructura.pdf $>$.

Guzmán, Carlota y Claudia Saucedo (2007), La voz de los estudiantes. Experiencias en torno a la escuela, México, Pomares.

Martín, A. (1998), "La departamentalización, contexto y concepto", Sinéctica, vol. 12, <http://www.redalyc.org/ pdf/998/99826006011.pdf> [Consulta: enero de 2019].

Loveless, Avril y Ben Williamson (2017), Nuevas identidades de aprendizaje en la era digital, Madrid, Narcea.

Meneses, E. (1971), "La organización departamental en las universidades", Revista Latinoamericana de Estudios Educativos, núm. 3, pp. 75-78.

Nicastro, Sandra y María Greco (2009), Entre trayectorias. Escenas y pensamientos en espacios de formación, Rosario, Argentina, Hommo Sapiens.

Rudduck, Jean y Julia Flutter (2007), Cómo mejorar tu centro escolar dando la voz al alumnado, Madrid, Morata. 
Torres, Jurjo (2011), La justicia curricular, Madrid, Morata.

Tójar, Juan Carlos (2006), Investigación cualitativa. Comprender y actuar, Madrid, Editorial La Muralla.

Universidad Nacional Autónoma de México (UNAM) (2015), Reglamento General para la presentación, aprobación, evaluación y modificación de planes de estudio, <http:// www.abogadogeneral.unam.mx/legislacion/abogen/ documento.html?doc_id=85>.

Wenger, E. (2001), Comunidades de práctica. Aprendizaje, significado e identidad, Barcelona, Paidós.

\section{Agradecimientos}

A la Dirección General de Asuntos del Personal Académico de la Universidad Nacional Autónoma de México, a través del proyecto PAPIME PE300217.

A las y los jóvenes estudiantes de Psicología que nos permitieron compartir sus voces en este artículo.

\section{Cómo citar este artículo:}

Díaz-Barriga Arceo, Frida, José-Luis López-Ramírez y Edmundo-Antonio López-Banda (2020), "Trayectorias personales de aprendizaje y currículo flexible: la perspectiva de los estudiantes universitarios de psicología", Revista Iberoamericana de Educación Superior (RIES), vol. XI, núm. 30, pp. 3-21, https://doi.org/10.22201/iisue.20072872e.2020.30.585 [Consulta: fecha de última consulta]. 